\title{
Joint Minimization of Uplink and Downlink Whole-Body Exposure Dose in Indoor Wireless Networks
}

\author{
D. Plets, ${ }^{1}$ W. Joseph, ${ }^{1}$ K. Vanhecke, ${ }^{1}$ G. Vermeeren, ${ }^{1}$ J. Wiart, ${ }^{2}$ S. Aerts, ${ }^{1}$ \\ N. Varsier, ${ }^{2}$ and L. Martens ${ }^{1}$ \\ ${ }^{1}$ Information Technology Department, Ghent University/iMinds, Gaston Crommenlaan 8, 9050 Ghent, Belgium \\ ${ }^{2}$ Orange Labs Networks and Carriers, 38-40 rue Général Leclerc, 92794 Issy Les Moulineaux, France \\ Correspondence should be addressed to D. Plets; david.plets@intec.ugent.be
}

Received 4 September 2014; Accepted 12 November 2014

Academic Editor: Francisco Falcone

Copyright (C) 2015 D. Plets et al. This is an open access article distributed under the Creative Commons Attribution License, which permits unrestricted use, distribution, and reproduction in any medium, provided the original work is properly cited.

\begin{abstract}
The total whole-body exposure dose in indoor wireless networks is minimized. For the first time, indoor wireless networks are designed and simulated for a minimal exposure dose, where both uplink and downlink are considered. The impact of the minimization is numerically assessed for four scenarios: two WiFi configurations with different throughputs, a Universal Mobile Telecommunications System (UMTS) configuration for phone call traffic, and a Long-Term Evolution (LTE) configuration with a high data rate. Also, the influence of the uplink usage on the total absorbed dose is characterized. Downlink dose reductions of at least $75 \%$ are observed when adding more base stations with a lower transmit power. Total dose reductions decrease with increasing uplink usage for WiFi due to the lack of uplink power control but are maintained for LTE and UMTS. Uplink doses become dominant over downlink doses for usages of only a few seconds for WiFi. For UMTS and LTE, an almost continuous uplink usage is required to have a significant effect on the total dose, thanks to the power control mechanism.
\end{abstract}

\section{Introduction}

The vast expansion of the use of wireless networks in everyday life has led to a greater awareness of exposure of the general public to RF (radio-frequency) electromagnetic fields used for wireless telecommunication. International organizations such as IEEE [1] and ICNIRP (International Commission on Non-Ionizing Radiation Protection) [2] have issued safety guidelines to limit the maximal electric-field strength due to wireless communications. Also, on a national level, authorities have implemented laws and norms to limit the exposure to electromagnetic fields. A lot of research has been done on the characterization of RF exposure (e.g., [3-7]), and measurements have indicated that exposure in indoor environments cannot be neglected [8].

Exposure studies mostly consider either the fields generated due to traffic from base station to user device (downlink) or exposure due to the electromagnetic waves induced in the body by the user device (uplink). Further, software tools for predicting the received signal quality [9-15] very often focus on Quality of Services parameters and do not account for exposure values. In [16], the authors presented the WiCa Heuristic Indoor Propagation Prediction (WHIPP) tool, a set of heuristic planning algorithms, experimentally validated for network planning in indoor environments [16]. The path loss prediction algorithm takes into account the effect of the environment on the wireless propagation channel and bases its calculations on the determination of the dominant path between transmitter and receiver, that is, the path along which the signal encounters the least obstruction. The WHIPP tool is designed for optimal network planning with a minimal number of access points (AP) [16]. In [17], this tool was extended for automatic network planning with limited or minimized downlink electric-field strength in indoor wireless networks, without impairing coverage. In [18], it was further extended with prediction algorithms to simulate and visualize electric-field strengths due to DL traffic and localized Specific Absorption Rate values in $10 \mathrm{~g}$ tissue $\left(\mathrm{SAR}_{10 \mathrm{~g}}\right)$ due to UL traffic. 
In this paper, instead of separating between UL (due to the mobile device's transmitted signal) and DL (due to the electric-fields $\mathrm{E}$ originating from the base stations or APs) traffic, exposure is spatially calculated as a whole-body dose due to both UL and DL [19]. Different optimization scenarios will be simulated using the WHIPP tool, and the impact on the whole-body dose will be numerically assessed. Additionally, the impact of the actual usage time on the dose reduction will be investigated. To the authors' knowledge, no indoor wireless network design solutions are yet available for the minimization of the total whole-body doses, where UL and DL contributions are both considered. Further, the impact of such redesign on the total exposure dose has not been quantified before, neither at specific locations in the building, nor globally over the entire building. Four scenarios (using WiFi, Universal Mobile Telecommunications System (UMTS), or Long-Term Evolution (LTE)) will be defined to investigate the influence of the number of indoor base stations, power control, and uplink transmission duration on the exposure.

In Section 2, the WHIPP tool used for minimization and assessment of exposure doses is discussed, the minimization metric (whole-body dose) is mathematically formulated, and the simulation scenarios are presented. In Section 3, the results for the different scenarios are presented and the impact of optimizing the network topology on the total dose is assessed, as well as the influence of the uplink usage on the total dose. Finally, conclusions and future work are presented in Section 4.

\section{Materials and Methods}

2.1. WHIPP Prediction Tool. The WHIPP algorithm is a heuristic planning algorithm, developed and validated for the prediction of path loss in indoor environments [16]. It takes into account the effect of the environment on the wireless propagation channel and has been developed for the prediction of the path loss on a grid over an entire building floor or at specific locations. The spatial granularity of the prediction is determined by the density of the grid points on the building floor. The algorithm bases its calculations on the determination of the dominant path between transmitter and receiver, that is, the path along which the signal encounters the lowest obstruction. This approach is justified by the fact that more than $95 \%$ of the energy received is contained in only 2 or 3 paths [11]. The dominant path is determined with a multidimensional optimization algorithm that searches the lowest total path loss, consisting of a distance loss (taking into account the length of the propagation path), a cumulated wall loss (taking into account the walls penetrated along the propagation path), and an interaction loss (taking into account the propagation direction changes of the path, e.g., around corners). The performance of the model has been validated with a large set of measurements in various buildings [16]. In contrast to many existing tools, no tuning of the tool's parameters is performed for the validation. Excellent correspondence between measurements and predictions is obtained, even for other buildings and floors [16]. The WHIPP tool contains a user interface that was developed in collaboration with usability experts. This allows visualizing not only path loss, throughput, or electricfield values, but, based on the formulations presented in the next section, also power densities, (localized or whole-body) absorption values, and doses.

2.2. Minimization Metric: Whole-Body Exposure Dose. The aim of the paper is to minimize the median whole-body absorbed dose $D_{\text {wb-total }}^{50}(\mathrm{~J} / \mathrm{kg})[19,20]$ over a given building floor where indoor base stations are installed. The total whole-body dose $D_{\text {wb-total }}$ at a certain location in a building is calculated as the sum of the whole-body dose $D_{\text {wb-DL }}(\mathrm{J} / \mathrm{kg})$ due to downlink and the whole-body dose $D_{\text {wb-UL }}(\mathrm{J} / \mathrm{kg})$ due to the user device's uplink:

$$
D_{\mathrm{wb}-\mathrm{total}}=D_{\mathrm{wb}-\mathrm{DL}}+D_{\mathrm{wb}-\mathrm{UL}}
$$

In the following sections, it will be explained how the downlink and uplink whole-body exposure doses are calculated.

2.2.1. Downlink Whole-Body Absorbed Dose $D_{w b-D L}$. To calculate $D_{\text {wb-DL }}(\mathrm{J} / \mathrm{kg})$, the whole-body SAR SAR $\mathrm{wb}_{\mathrm{DL}}(\mathrm{W} / \mathrm{kg})$ due to downlink is multiplied by $T_{\text {total }}(\mathrm{s})$; the time duration of the exposure

$$
D_{\text {wb-DL }}=T_{\text {total }} \cdot \mathrm{SAR}_{\mathrm{wb}-\mathrm{DL}}
$$

SAR $_{\text {wb-DL }}$ accounts for the downlink exposure due to all base stations and is calculated as follows:

$$
\mathrm{SAR}_{\mathrm{wb}-\mathrm{DL}}=\sum_{\mathrm{BS}_{i}}\left(S_{\mathrm{BS}_{i}} \cdot \mathrm{SAR}_{\mathrm{REF}_{\mathrm{wb}}}^{\mathrm{DL}_{\mathrm{BAT}}}\right)
$$

where $S_{\mathrm{BS}_{i}}\left(\mathrm{~W} / \mathrm{m}^{2}\right)$ is the received power density due to base

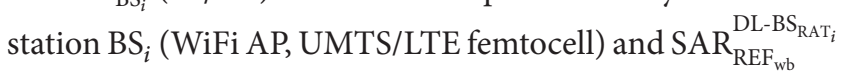
$\left(\mathrm{W} / \mathrm{kg}\right.$ per $\left.\mathrm{W} / \mathrm{m}^{2}\right)$ is the reference whole-body SAR (for $1 \mathrm{~W} / \mathrm{m}^{2}$ of received power density) due to $\mathrm{BS}_{i}$ using a certain Radio Access Technology (RAT). Power densities from RATs using different frequencies will contribute to $\mathrm{SAR}_{\text {wb-DL }}$ according to the reference whole-body SAR for the RAT at that frequency. Therefore, (3) sums over the power densities from each of the base stations $\mathrm{BS}_{i}$. The power density $S_{\mathrm{BS}_{i}}\left(\mathrm{~W} / \mathrm{m}^{2}\right)$ is related to the electric-field strength as follows:

$$
S_{\mathrm{BS}_{i}}=\frac{E_{\mathrm{BS}_{i}}^{2}}{Z_{0}}=\frac{E_{\mathrm{BS}_{i}}^{2}}{120 \cdot \pi}=\frac{E_{\mathrm{BS}_{i}}^{2}}{377},
$$

where $E_{\mathrm{BS}_{i}}(\mathrm{~V} / \mathrm{m})$ is the electric-field strength due to base station $\mathrm{BS}_{i}$, observed at the considered location and with an assumed duty cycle of $100 \% . Z_{0}$ is the free-space impedance, equal to $377 \Omega$. For WiFi, the actual duty cycle DC [-] of the traffic generated by $\mathrm{BS}_{i}[21]$ must also be accounted for, since it represents the relative transmission time of a signal. In WiFi, signals are not transmitted continuously and therefore the predicted power densities at $100 \%$ operation need to be multiplied by the duty cycle. For UMTS and LTE, the duty 
cycle is $100 \%$ for downlink. When accounting for the duty cycle, (4) can be rewritten as follows:

$$
S_{\mathrm{BS}_{i}}=\frac{E_{\mathrm{BS}_{i}}^{2} \cdot \mathrm{DC}}{377} \text {. }
$$

2.2.2. Uplink Whole-Body Absorbed Dose $D_{w b-U L}$. To calculate $D_{\text {wb-UL }}(\mathrm{J} / \mathrm{kg})$, the whole-body SAR SAR ${ }_{\text {wb-UL }}(\mathrm{W} / \mathrm{kg})$ due to uplink is multiplied by $T_{\text {usage }}(\mathrm{s})$, the time duration of the usage. $T_{\text {usage }}$ is a value between 0 and $T_{\text {total }}$ :

$$
D_{\text {wb-UL }}=T_{\text {usage }} \cdot \mathrm{SAR}_{\mathrm{wb}-\mathrm{UL}} \cdot
$$

SAR $_{\text {wb-UL }}$ is the SAR due to the UL traffic from the mobile device towards base station $\mathrm{BS}_{c}$ it is connected to, using a certain RAT. It is calculated as follows:

$$
\mathrm{SAR}_{\mathrm{wb}-\mathrm{UL}}=P_{\mathrm{BS}_{c}}^{\mathrm{Tx}} \cdot \mathrm{DC} \cdot \mathrm{SAR}_{\mathrm{REF}_{\mathrm{wb}}}^{\mathrm{UL}_{\mathrm{RAT}}},
$$

where $P_{\mathrm{BS}}^{\mathrm{Tx}}(\mathrm{W})$ is the mobile device's power transmitted towards the base station $\mathrm{BS}_{c}$ it is connected to, DC [-] is again the WiFi duty cycle of the UL traffic, and $\mathrm{SAR}_{\mathrm{REF}_{\mathrm{wb}}}^{\mathrm{UL}_{\mathrm{RAT}}}(\mathrm{W} / \mathrm{kg}$ per $\mathrm{W}$ ) is the reference whole-body SAR (for $1 \mathrm{~W}$ of transmitted power) due to the mobile device operating at RAT. For UMTS and LTE, the duty cycle is $100 \%$ for uplink.

In future research, also whole-body absorption due to the uplink transmission of other users will be accounted for.

2.2.3. Input Parameters. The equations formulated above now allow calculating absorbed doses. However, some of the parameters are required as input or need to be calculated by the WHIPP tool.

(i) In (5), $E_{\mathrm{BS}_{i}}(\mathrm{~V} / \mathrm{m})$ (electric-field strength due to base station $\mathrm{BS}_{i}$ ) can be calculated by the WHIPP tool as described in $[17,18,22]$, where a far-field conversion formula between path loss and electric-field strength is presented:

$$
\begin{aligned}
\mathrm{PL}(\mathrm{dB})= & 139-E_{\mathrm{ERP}=1 \mathrm{~kW}}(\mathrm{~dB} \mu \mathrm{V} / \mathrm{m}) \\
& +20 \cdot \log _{10}(f)(\mathrm{MHz}),
\end{aligned}
$$

with PL $(\mathrm{dB})$ as the path loss between the transmitter and a receiver at a certain location, $E_{\mathrm{ERP}=1 \mathrm{~kW}}$ $(\mathrm{dB} \mu \mathrm{V} / \mathrm{m})$ as the received field strength for an ERP (Effective Radiated Power) of $1 \mathrm{~kW}$, and $f(\mathrm{MHz})$ as the frequency.

Using (8) and the identity

$$
E(\mathrm{~V} / \mathrm{m})=E_{\mathrm{ERP}=1 \mathrm{~kW}}(\mathrm{~V} / \mathrm{m}) \cdot \sqrt{\operatorname{ERP}(\mathrm{kW})},
$$

and knowing that for dipoles ERP $(\mathrm{dBm})=\mathrm{EIRP}$ $(\mathrm{dBm})-2.15$, we obtain the following formula for the electric-field strength $E_{\mathrm{BS}_{i}}(\mathrm{dBV} / \mathrm{m})$ at a certain location, as a function of the $\operatorname{EIRP}_{\mathrm{BS}_{i}}(\mathrm{dBm})$ of the base station, the path loss, and the base station's frequency $f_{\mathrm{BS}_{i}}(\mathrm{MHz})$ :

$$
E_{\mathrm{BS}_{i}}(\mathrm{dBV} / \mathrm{m})=\mathrm{EIRP}_{\mathrm{BS}_{i}}-43.15+20 \cdot \log _{10}\left(f_{\mathrm{BS}_{i}}\right)-\mathrm{PL}
$$

or, with $E_{\mathrm{BS}_{i}}$ expressed in $(\mathrm{V} / \mathrm{m})$,

$$
E_{\mathrm{BS}_{i}}(\mathrm{~V} / \mathrm{m})=10^{\left(\mathrm{EIRP}_{\mathrm{BS}_{i}}-43.15+20 \cdot \log _{10}\left(f_{\mathrm{BS}_{i}}\right)-\mathrm{PL}\right) / 20} .
$$

PL $(\mathrm{dB})$ is here predicted by the WHIPP tool [16].

(ii) The duty cycle (in (5) and (7)) depends on the type and amount of traffic over the air [21]. In the following sections, simplified duty cycle value assumptions will be made, depending on the considered network topology.

(iii) In (7), $P_{\mathrm{BS}}^{\mathrm{Tx}}(\mathrm{dBm})$ (mobile device's power transmitted towards the base station BS it is connected to) for phone call connections with a UMTS femtocell will be calculated with the WHIPP tool as described in [18]:

$$
P_{\mathrm{BS}_{c}}^{\mathrm{Tx}}=P_{\text {sens }}+\mathrm{PL},
$$

where $P_{\text {sens }}(\mathrm{dBm})$ is the sensitivity of the UMTS femtocell base station for maintaining a UMTS phone call, determined and validated at $-110 \mathrm{dBm}$ [18]. PL $(\mathrm{dB})$ is again the path loss between transmitter and receiver locations, as calculated by the WHIPP tool [16]. Thanks to power control, $P_{\mathrm{BS}_{c}}^{\mathrm{Tx}}$ values will be lower for good connections with the base stations (low PL). The lower and upper limits for $P_{\mathrm{BS}}^{\mathrm{Tx}}$ values are -57 and $23 \mathrm{dBm}$, respectively (see also [23]).

For LTE, $P_{\mathrm{BS}}^{\mathrm{Tx}}(\mathrm{dBm})$ will be modeled as described in $[24,25]$ :

$$
P_{\mathrm{BS}_{c}}^{\mathrm{Tx}}=P_{0 \_ \text {pusch }}+\alpha \mathrm{PL}+10 \log (M)+\delta,
$$

with $P_{0 \text { _pusch }}$ as the required received power at the femtocell base station (FBS) over a bandwidth of one resource block, $\alpha$ as a path-loss compensation factor, $M$ as the number of resource blocks being used, and $\delta$ as a network-controlled factor, reflecting power increase or decrease commands. $\alpha$ will be assumed equal to 1 and $\delta$ equal to zero. For the $20-\mathrm{MHz}$ channel used in this paper, $M$ is equal to 100 . For the considered indoor environment, $P_{0_{-} \text {pusch }}$ is set equal to $-96 \mathrm{dBm}$ [25]. The lower and upper limits for $P_{\mathrm{BS}_{c}}^{\mathrm{Tx}}$ values are -40 and $23 \mathrm{dBm}$, respectively [24].

For WiFi, no power control is used and a fixed value of $20 \mathrm{dBm}$ for $P_{\mathrm{BS}_{c}}^{\mathrm{Tx}}$ is assumed.

(iv) The reference $S A R$ values for $1 \mathrm{~W} / \mathrm{m}^{2}$ observed power density from (3) and for $1 \mathrm{~W}$ transmitted power from (7) are listed in Table 1 for the three considered RATs. The downlink whole-body reference SAR values for UMTS and WiFi are obtained from [20]. As human model, the Duke model of the Virtual Family was used [26]. It is generated from a set of magnetic resonance images of whole-body scans from a 34-yearold male (height of $1.74 \mathrm{~m}$, weight of $72 \mathrm{~kg}$, and body mass index of $23.1 \mathrm{~kg} / \mathrm{m}$ ). Since the LTE downlink frequency band $(2.6 \mathrm{GHz})$ is close to the WiFi band 
TABLE 1: Reference whole-body SAR values $\mathrm{SAR}_{\mathrm{REF}}^{\mathrm{DL}-\mathrm{S}_{\mathrm{wAT}}}$ and $S_{A R} \mathrm{RLF}_{\mathrm{Rb}} \mathrm{L}_{\mathrm{RAT}}$ for WiFi and UMTS, expressed in $\mathrm{W} / \mathrm{kg}$ per $1 \mathrm{~W}$ transmitted power (UL) or in $\mathrm{W} / \mathrm{kg}$ per $1 \mathrm{~W} / \mathrm{m}^{2}$ observed power density (DL).

\begin{tabular}{lccc}
\hline RAT & $\begin{array}{c}\text { Frequency } \\
(\mathrm{MHz})\end{array}$ & $\begin{array}{c}\mathrm{SAR}_{\mathrm{REF}}^{\mathrm{DL}-\mathrm{BS}_{\mathrm{RAT}}} \\
\left(\mathrm{W} / \mathrm{kg} \text { per W/m } / \mathrm{m}^{2}\right)\end{array}$ & $\begin{array}{c}\mathrm{SAR}_{\mathrm{REF}}^{\mathrm{UL}_{\mathrm{RT}}} \\
(\mathrm{W} / \mathrm{kg} \text { per W })\end{array}$ \\
\hline UMTS & 1950 & 0.003 & 0.00495 \\
WiFi & 2400 & 0.0028 & 0.0070 \\
LTE & 2600 & 0.0028 & 0.0070 \\
\hline
\end{tabular}

$(2.4 \mathrm{GHz})$, it is fair to assume the same value for LTE as for WiFi. The uplink whole-body reference SAR values for UMTS are also obtained from [20] (cell phone placed to the right side of the head of the human model). The whole-body reference SAR value $\mathrm{SAR}_{\mathrm{REF}_{\mathrm{wb}}}^{\mathrm{UL}_{\mathrm{RAT}}}$ values for WiFi and LTE for data usage are obtained through Finite-Difference Time-Domain (FDTD) simulations, where the mobile device is held in front of the body. The resolution of the human model was chosen to be $2 \mathrm{~mm} \times 2 \mathrm{~mm} \times 2 \mathrm{~mm}$, resulting in a total of about 110 million voxels [26]. The same mobile phone is assumed as in [18].

2.2.4. Optimization Algorithm. In [17], an automatic electricfield minimization algorithm was designed, providing a userdefined throughput while ensuring a low and homogeneous field strength. It is based on the creation of networks consisting of low-power access points. This not only lowers the downlink exposure but, for technologies using power control mechanisms, also reduces uplink exposure thanks to the higher probability of access points being located near the user device. In the following, four scenarios will be defined to assess the impact of low-exposure indoor wireless deployments on the uplink, downlink, and total whole-body exposure doses. For each scenario, the wireless networks will be designed based on the receiver sensitivities corresponding to the envisioned throughput.

2.3. Scenarios. Two WiFi scenarios with different data requirements, one UMTS femtocell phone call scenario and one LTE femtocell data scenario, will be investigated. All scenarios are investigated in the office building depicted in Figure 1. The building is $90 \mathrm{~m}$ long and $17 \mathrm{~m}$ wide and consists of concrete walls (grey) and layered drywalls (brown). For all scenarios, a receiver height of $130 \mathrm{~cm}$ above ground level is assumed. For the design of the networks, a shadowing margin of $7 \mathrm{~dB}$ and a fading margin $5 \mathrm{~dB}$ are assumed [16].

\subsubsection{Scenario 1: WiFi Deployment for 54 Mbps: Device in} front of Body. In the first scenario, two WiFi configurations are compared. The configurations are the ones from [17], in which a traditional network deployment (with maximalpower Equivalent Isotropically Radiated Powers (EIRPs)) and an exposure-optimized network deployment are compared based on only their electric-field strength distributions and

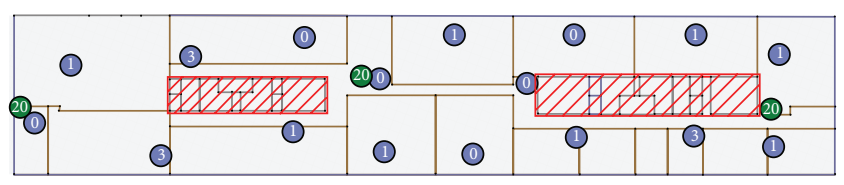

(a)

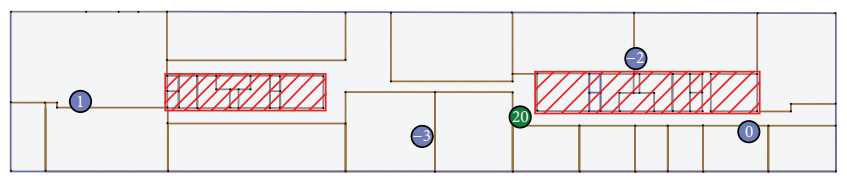

(b)

FIgURE 1: WiFi network configurations for the traditional deployment (green APs with EIRP $=20 \mathrm{dBm}$ ) and for the exposureoptimized configuration (purple APs with EIRP between -3 and $1 \mathrm{dBm}$ ) for (a) $54 \mathrm{Mbps}$ and (b) $18 \mathrm{Mbps}$. EIRP in $\mathrm{dBm}$ is indicated within dots.

based on a worst-case scenario with a duty cycle of $100 \%$. The exposure-optimized scenario aims for a minimization of the median and the $95 \%$ percentile of the field values observed on the building floor [17]. Both deployments were designed to provide a high throughput (54 Mbps), corresponding to a receiver sensitivity of $-68 \mathrm{dBm}$ for an $802.11 \mathrm{~b} / \mathrm{g}$ reference receiver [17]. Figure 1(a) shows the two network deployments. The traditional network deployment consists of the three 20 $\mathrm{dBm}$ APs (green) and the exposure-optimized deployment consists of the 17 low-EIRP APs (purple). The locations where no coverage is required (kitchen, toilet, shed, elevator, etc.) are shaded. For the downlink duty cycles, the assumption explained in [21] will be used. In the exposure-optimized configuration, the same amount of users is served by 5.66 (17/3) times more APs or one AP needs to serve 5.66 times less users. We assume that the downlink duty cycle for each AP is 3\% for the exposure-optimized deployment and 17\% (5.66 times 3\%) for the traditional configuration. The uplink duty cycle will be assumed to be $2 \%$, irrespective of the network configuration (duty cycles from [21]).

2.3.2. Scenario 2: WiFi Deployment for $18 \mathrm{Mbps}$ : Device in front of Body. In the second scenario, a traditional and an exposure-optimized deployment are designed for a throughput of $18 \mathrm{Mbps}$, corresponding to a receiver sensitivity of $-82 \mathrm{dBm}$ [17]. Figure 1(b) shows the two network deployments for scenario 2. The traditional network deployment consists of one $20 \mathrm{dBm}$ green AP and the exposure-optimized deployment consists of the 4 low-EIRP purple APs. The locations where no coverage is required are again shaded. For the downlink duty cycles, the assumption explained in [21] will again be used. Compared to the traditional deployment in scenario 1, one AP is used instead of three and a duty cycle of $51 \%$ (3 times 17\%) is assumed for the traditional deployment. The exposure-optimized deployment has four APs, so a duty cycle of $12.75 \%$ (51 divided by 4 ) is assumed. The uplink duty cycle will again be assumed to be $2 \%$, irrespective of the network configuration (duty cycles from [21]). 


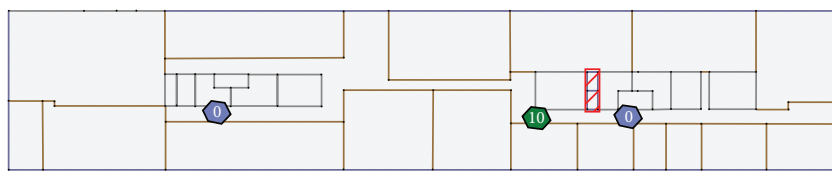

(a)

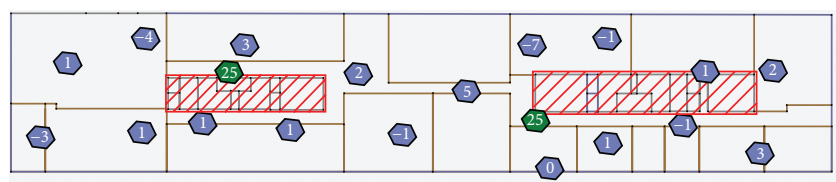

(b)

FIGURE 2: (a) UMTS network configurations for the traditional deployment (green FBSs with EIRP $=10 \mathrm{dBm}$ ) and for the exposureoptimized configuration (purple FBSs with EIRP of $0 \mathrm{dBm}$ ) for voice call connections and (b) LTE network configurations for the traditional deployment (green FBSs with EIRP $=25 \mathrm{dBm}$ ) and for the exposure-optimized configuration (purple FBSs with EIRP between -7 and $5 \mathrm{dBm}$ ) for $36 \mathrm{Mbps}$. EIRP in $\mathrm{dBm}$ is indicated within dots.

2.3.3. Scenario 3: UMTS Deployment for Voice Calls: Device to Side of Head. In the third scenario, a traditional and an exposure-optimized UMTS femtocell deployment are compared. The traditional deployment uses 1 UMTS femtocell base station (FBS) with an EIRP of $10 \mathrm{dBm}$ to cover the entire building floor for phone call coverage, corresponding to a receiver sensitivity of $-95.1 \mathrm{dBm}$ [18]. The exposureoptimized deployment covers the floor with two UMTS FBSs with an EIRP of $0 \mathrm{dBm}$. In this UMTS scenario, the configuration with more FBSs (with a lower EIRP) will have the additional benefit of a lower average device transmitted power due to power control, whereas for WiFi the transmittance power of the mobile phone is fixed. Figure 2 shows the two network deployments in the top figure. The traditional network deployment consists of one $10 \mathrm{dBm}$ green FBS and the exposure-optimized deployment consists of two $0 \mathrm{dBm}$ FBSs (purple). In the elevators, no phone call coverage is required. These areas are shaded.

2.3.4. Scenario 4: LTE Deployment for $40.6 \mathrm{Mbps}$ : Device in front of Body. In the fourth scenario, a traditional and an exposure-optimized LTE femtocell deployment for a throughput of $40.6 \mathrm{Mbps}$ are designed. According to [17], for a $20 \mathrm{MHz}$ channel, this corresponds to a required received power of $-68.1 \mathrm{dBm}$ [17]. Figure 2 shows the two network deployments in the bottom figure. In the traditional network deployment, the two green LTE FBSs with an EIRP of $25 \mathrm{dBm}$ are sufficient to provide the required coverage, while the exposure-optimized deployment covers the floor with the 18 purple LTE FBSs with an EIRP between -7 and $5 \mathrm{dBm}$. In the shaded areas, no coverage is required. Exposure optimization clearly leads to a higher number of base stations and thus also to a higher cost.

\section{Results and Discussion}

3.1. Scenario 1: WiFi: 54 Mbps. Figure 3 shows the cumulative distribution function (cdf) of the whole-body doses over

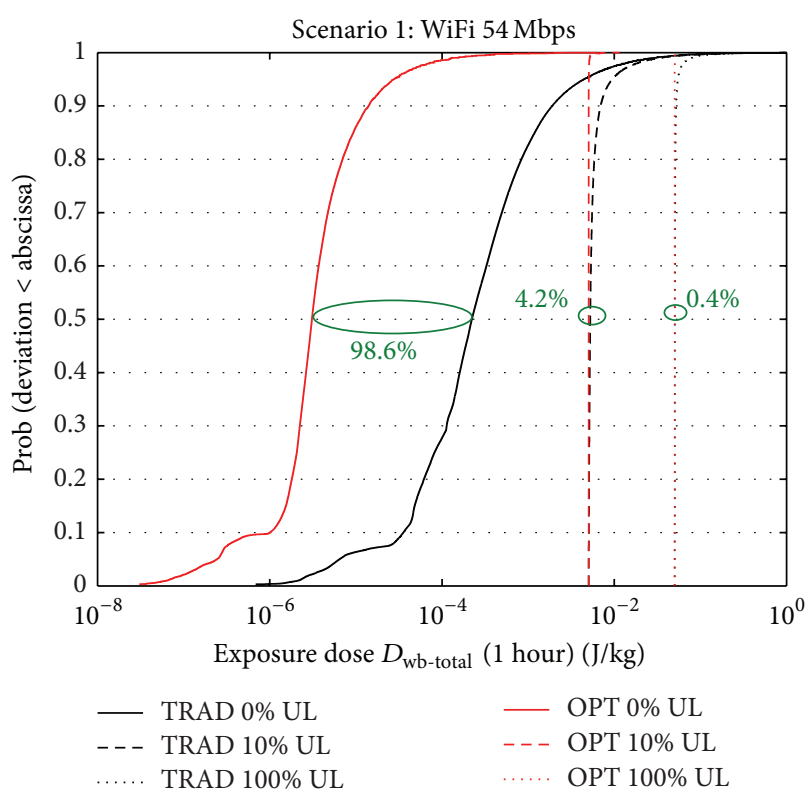

Figure 3: CDF of total dose (uplink + downlink) within one hour for scenario 1 for traditional and optimized deployment for three uplink usages $(0 \%, 10 \%$, and $100 \%$ of the time).

a time frame of one hour for the traditional and optimized deployment for three uplink usages $(0 \%, 10 \%$, and $100 \%$ of the time) for WiFi with a downlink throughput of $54 \mathrm{Mbps}$. It also shows the median whole-body dose reductions when switching from a traditional to an exposure-optimized configuration. The mobile device is held in front of the body. The " $0 \%$ UL" cdfs correspond with the DL-only dose. Table 2 shows the $50 \%$ and $95 \%$ percentile of the doses for the different configurations of scenario 1. Figure 3 and Table 2 show that the downlink dose ( $0 \%$ UL) is drastically lowered in the exposure-optimized deployment (higher number of BS with a lower EIRP): a reduction of the median $\left(D_{\text {wb-total }}^{50}\right)$, from $2.2 \cdot 10^{-4}$ to $3.1 \cdot 10^{-6} \mathrm{~J} / \mathrm{kg}$ (reduction of $98.6 \%$ ) and a reduction of $99.4 \%$ for the $95 \%$ percentile $\left(D_{\mathrm{wb} \text {-total }}^{95}\right)$ of the total dose. For the WiFi scenario, both configurations (traditional and optimized) will cause the same uplink powers and uplink doses due to the absence of power control in WiFi devices: irrespective of the connection quality with the AP, a fixed power of $20 \mathrm{dBm}$ is assumed. Figure 3 and Table 2 show that, as the uplink usage increases (from 0 to 10 to $100 \%$ ), the total dose is becoming quickly dominated by the uplink dose. For example, when comparing a usage of $10 \%$ with DL-only $(0 \%$ usage), the median of the total dose $\left(D_{\text {wb-total }}^{50}\right)$ is 24 times higher for the traditional deployment $(0.0053$ versus 2.22 . $\left.10^{-4}\right)$ and 1618 times higher for the optimized deployment $\left(0.005\right.$ versus $\left.3.09 \cdot 10^{-6}\right)$. The increasing dominance of the uplink causes the median dose reductions to become gradually smaller: from $98.6 \%$ for DL-only to $4.2 \%$ and $0.4 \%$ for $10 \%$ and $100 \%$ usage, respectively (see $\%$ RED in Table 2 and in Figure 3). The small dose reductions for an UL usage of $100 \%$ correspond to the almost coinciding plots for the traditional and optimized configurations. Unlike the median, 
TABLE 2: 50\% and 95\% percentile values of total dose ( $/ \mathrm{kg}$ ) for four scenarios for traditional and optimized configurations for different uplink usages $(0 \%, 10 \%$, and $100 \%)$ and dose reduction $(\%)$. (TRAD = traditional deployment, OPT = optimized deployment, and $\%$ RED $=$ exposure dose reduction percentage when switching from TRAD to OPT).

\begin{tabular}{|c|c|c|c|c|c|c|c|c|c|}
\hline \multirow{3}{*}{ Total dose (J/kg) } & \multicolumn{9}{|c|}{ Uplink usage } \\
\hline & \multicolumn{3}{|c|}{$0 \% \mathrm{UL}$} & \multicolumn{3}{|c|}{$10 \% \mathrm{UL}$} & \multicolumn{3}{|c|}{$100 \% \mathrm{UL}$} \\
\hline & TRAD & OPT & $\%$ RED & TRAD & OPT & $\%$ RED & TRAD & OPT & $\%$ RED \\
\hline \multicolumn{10}{|l|}{ Scenario 1} \\
\hline$D_{\text {wb-total }}^{50}$ & $2.22 \cdot 10^{-4}$ & $3.09 \cdot 10^{-6}$ & 98.6 & 0.0053 & 0.005 & 4.2 & 0.0506 & 0.0504 & 0.4 \\
\hline$D_{\text {wb-total }}^{95}$ & 0.0045 & $2.76 \cdot 10^{-5}$ & 99.4 & 0.0095 & 0.0051 & 46.6 & 0.0549 & 0.0504 & 8.1 \\
\hline \multicolumn{10}{|l|}{ Scenario 2} \\
\hline$D_{\text {wb-total }}^{50}$ & $1.85 \cdot 10^{-5}$ & $6.77 \cdot 10^{-7}$ & 96.3 & 0.0051 & 0.005 & 0.4 & 0.0504 & 0.0504 & 0.04 \\
\hline$D_{\text {wb-total }}^{95}$ & 0.0012 & $1.54 \cdot 10^{-5}$ & 98.7 & 0.0062 & 0.0051 & 18.6 & 0.0516 & 0.0504 & 2.2 \\
\hline \multicolumn{10}{|l|}{ Scenario 3} \\
\hline$D_{\text {wb-total }}^{50}$ & $3.72 \cdot 10^{-6}$ & $9.35 \cdot 10^{-7}$ & 74.9 & $4.53 \cdot 10^{-6}$ & $1.18 \cdot 10^{-6}$ & 74.0 & $1.28 \cdot 10^{-5}$ & $3.46 \cdot 10^{-6}$ & 72.9 \\
\hline$D_{\text {wb-total }}^{95}$ & $2.19 \cdot 10^{-4}$ & $4.11 \cdot 10^{-5}$ & 81.2 & $2.39 \cdot 10^{-4}$ & $4.45 \cdot 10^{-5}$ & 81.4 & $2.68 \cdot 10^{-4}$ & $4.45 \cdot 10^{-5}$ & 83.4 \\
\hline \multicolumn{10}{|l|}{ Scenario 4} \\
\hline$D_{\text {wb-total }}^{50}$ & $5.21 \cdot 10^{-4}$ & $1.02 \cdot 10^{-4}$ & 80.5 & $1.80 \cdot 10^{-3}$ & $1.28 \cdot 10^{-4}$ & 92.7 & $7.00 \cdot 10^{-3}$ & $2.75 \cdot 10^{-4}$ & 96.1 \\
\hline$D_{\text {wb-total }}^{95}$ & $1.39 \cdot 10^{-2}$ & $8.95 \cdot 10^{-4}$ & 93.6 & $1.63 \cdot 10^{-2}$ & $9.66 \cdot 10^{-4}$ & 94.1 & $4.17 \cdot 10^{-2}$ & $2.60 \cdot 10^{-3}$ & 93.8 \\
\hline
\end{tabular}

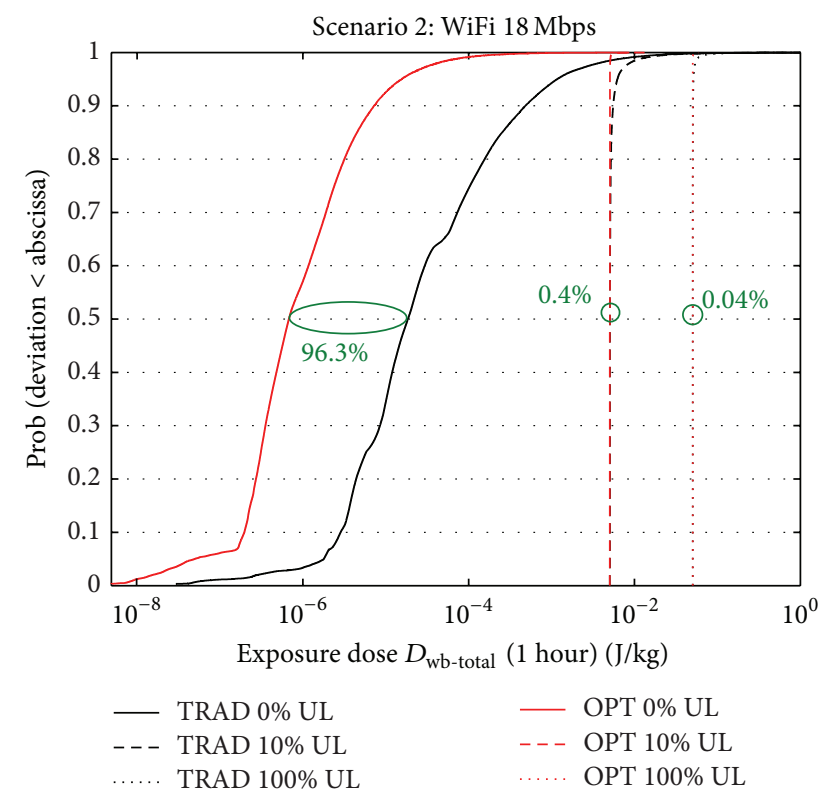

Figure 4: CDF of total dose (uplink + downlink) within one hour for scenario 2 for traditional and optimized deployment for three uplink usages $(0 \%, 10 \%$, and $100 \%$ of the time).

the $95 \%$ percentile (highest doses) is still significantly reduced $(46.6 \%)$ when using the optimized configuration with a $10 \%$ UL usage.

3.2. Scenario 2: WiFi: $18 \mathrm{Mbps}$. Figure 4 shows the cdf of the doses over a time frame of one hour for the traditional and optimized deployment for three uplink usages $(0 \%, 10 \%$, and $100 \%$ of the time), now for a WiFi throughput of $18 \mathrm{Mbps}$. It again shows the median whole-body dose reductions when switching from a traditional to an exposure-optimized configuration. Similarly, as in scenario 1, the downlink dose
( $0 \% \mathrm{UL})$ is drastically lowered when deploying more (lowerpower) base stations (exposure-optimized) instead of fewer base stations with a higher EIRP (traditional): the reductions equal $96.3 \%$ and $98.7 \%$ for median and 95\% percentile, respectively. Due to the lack of power control in WiFi, the total dose is again being dominated by the uplink dose for higher uplink usages, even more than that for scenario 1 . When comparing a usage of $10 \%$ with DL-only ( $0 \%$ usage), the median of the total dose is 276 times higher for the traditional deployment $\left(0.0051\right.$ versus $\left.1.85 \cdot 10^{-5} \mathrm{~J} / \mathrm{kg}\right)$ and 7385 times higher for the optimized deployment $(0.005$ versus $6.77 \cdot 10^{-7} \mathrm{~J} / \mathrm{kg}$ ), indicating that the DL dose quickly becomes negligible compared to the UL dose. Thanks to the lower throughput requirement, the DL doses are lower than those for scenario 1 (Table 2, $0 \% \mathrm{UL}$, scenario 1 versus scenario 2 ), indeed causing the UL dose (which is the same as that for scenario 1) to become more dominant. This is reflected by the lower reductions for the optimized configuration, for example, $0.4 \%$ (median) and $18.6 \%$ (95\% percentile) for $10 \%$ usage in scenario 2, compared to $4.2 \%$ (median) and $46.6 \%$ (95\% percentile) in scenario 1 (see \%RED in Table 2 and in Figure 4).

3.3. Scenario 3: UMTS: Voice Calls. Figure 5 shows the cdf of the doses over a time frame of one hour for the traditional and optimized deployment for three uplink usages $(0 \%, 10 \%$, and $100 \%$ of the time) for the UMTS voice call scenario, where the mobile device is held to the right side of the head. Again, a significant reduction is noticed in the total dose when deploying the optimized network. DL reductions are smaller than those for scenarios 1 and 2, due to the lower EIRP of the UMTS femtocell in the traditional deployment $(10 \mathrm{dBm}$ versus $20 \mathrm{dBm})$. However, total downlink doses are still reduced by $74.9 \%$ and $81.2 \%$ for median and $95 \%$ percentile, respectively (see \%RED in Table 2 and Figure 5).

In addition to a lower DL dose and unlike for $\mathrm{WiFi}$, the exposure-optimized deployment with more base stations also 


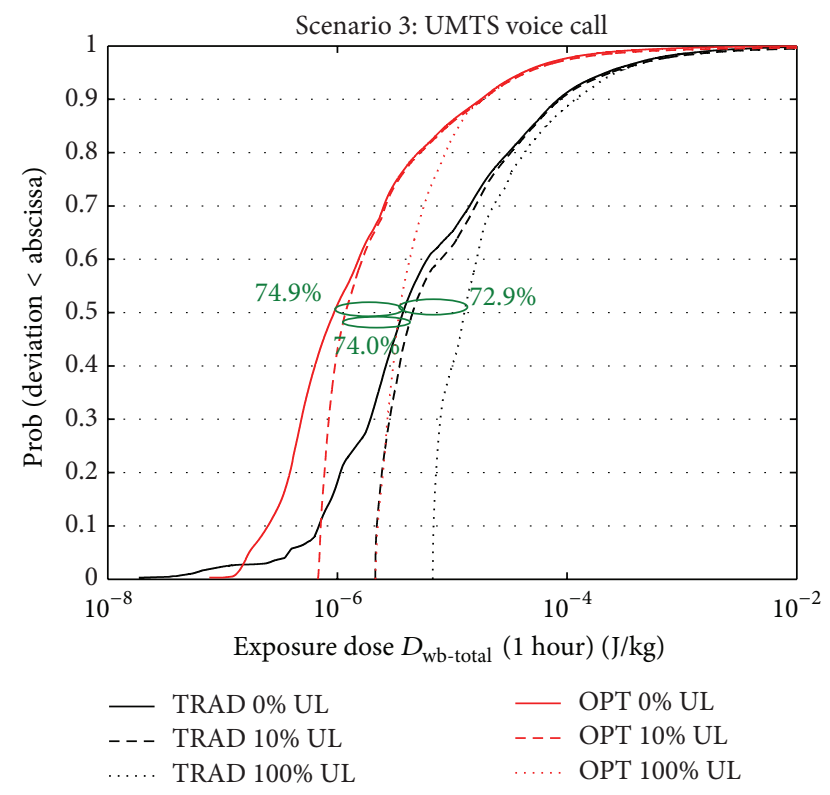

FIgURE 5: CDF of total dose (uplink + downlink) within one hour in an adult man for scenario 3 for traditional and optimized deployment for three uplink usages $(0 \%, 10 \%$, and $100 \%$ of the time).

allows taking advantage of the power control mechanism in UMTS. Due to the higher number of base stations, the mobile phone will-on average-require a lower transmittance power to maintain its connection. Figure 5 and Table 2 show that, for increasing UL usages, the dose reductions are maintained. The reduction of the median dose for $10 \%$ and $100 \%$ UL usage is $74.0 \%$ and $72.9 \%$, respectively, compared to $74.9 \%$ for DL-only. Also, for high UL usages, the UL dose does not become dominant over the DL dose: for the traditional deployment and for an uplink usage of $100 \%$, the UL whole-body dose is only 2.4 times higher than the DL dose: $9.08 \cdot 10^{-6} \mathrm{~J} / \mathrm{kg}$ (" $100 \%$ UL total dose" minus " $0 \%$ UL total dose") versus $3.72 \cdot 10^{-6} \mathrm{~J} / \mathrm{kg}$. For the optimized deployment, the UL dose is 2.7 times higher. Figure 5 and Table 2 show that even when calling the entire time $(100 \%$ UL usage), the whole-body total dose for the optimized deployment $\left(3.46 \cdot 10^{-6} \mathrm{~J} / \mathrm{kg}\right)$ still remains below the wholebody total dose for the traditional deployment without UL usage $\left(3.72 \cdot 10^{-6} \mathrm{~J} / \mathrm{kg}\right)$.

Figure 6 shows the total whole-body dose (in $\mu \mathrm{J} / \mathrm{kg}$ ) in a one-hour period in an adult man ("Duke," see Section 2.2.3) when calling the entire hour, for the two configurations: traditional (top) and optimized (bottom). The traditional deployment shows that the total dose is highly close to the FBS (high downlink dose) and near the cell edges (high uplink dose). For the optimized deployment, locations close to the FBS have lower downlink doses, thanks to the lower FBS EIRP, and locations far from the FBS have lower uplink doses thanks to the presence of the additional FBS. Figure 6 clearly shows that the high doses (lighter colors) are lowered for the optimized deployment.

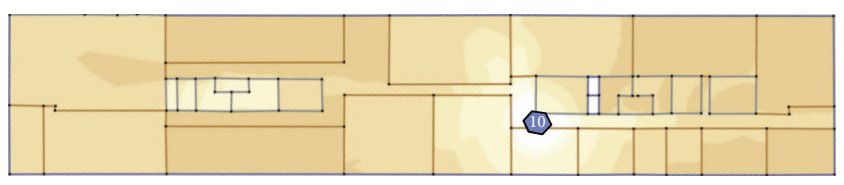

(a)

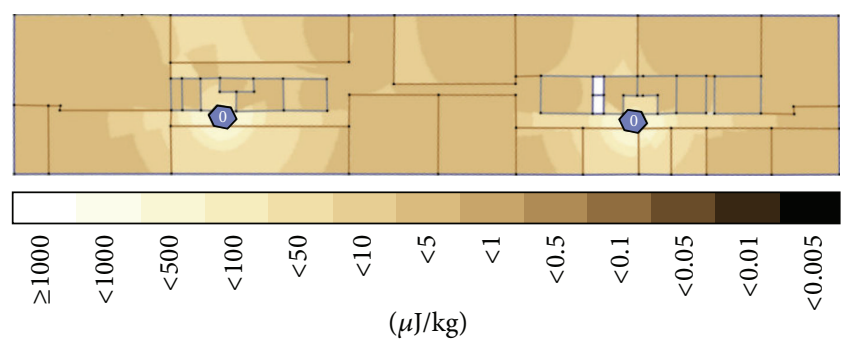

(b)

FIGURE 6: $D_{\text {wb-total }}$ distribution for a one-hour period when calling the entire hour for traditional deployment (top) and optimized deployment (bottom). FBS EIRP in $\mathrm{dBm}$ is indicated within hexagon.

3.4. Scenario 4: LTE: $40.6 \mathrm{Mbps}$. Figure 7 shows the cdf of the doses over a time frame of one hour for traditional and optimized deployment for three uplink usages $(0 \%, 10 \%$, and $100 \%$ of the time), for the LTE data scenario. The mobile device is again held in front of the body. Similarly to the previous scenarios, strong reductions of at least $80 \%$ are obtained, irrespective of the uplink usage. Analogously to UMTS, LTE also benefits from the power control mechanism. However, compared to UMTS, UL doses for LTE can become slightly more dominant over DL doses: for an uplink usage of $100 \%$, the UL whole-body dose is 12 times higher than the DL dose for the traditional deployment $\left(6.5 \cdot 10^{-3}\right.$ versus $5.21 \cdot 10^{-4} \mathrm{~J} / \mathrm{kg}$ ) and 1.7 times for the optimized deployment $\left(1.73 \cdot 10^{-4}\right.$ versus $\left.1.02 \cdot 10^{-4} \mathrm{~J} / \mathrm{kg}\right)$. Indeed, for LTE, the optimized deployment is more beneficial for uplink dose reduction: as the UL usage increases, higher dose reductions are observed; for example, median dose reduction increases from $80.5 \%$ to $96.1 \%$ when uplink usage increases from $0 \%$ to $100 \%$. For $100 \%$ uplink usage, the total dose for the optimized deployment $\left(2.75 \cdot 10^{-5} \mathrm{~J} / \mathrm{kg}\right)$ is a factor 19 smaller than the total dose for the traditional deployment without UL usage $\left(5.21 \cdot 10^{-4} \mathrm{~J} / \mathrm{kg}\right)$.

3.5. Comparison of Scenarios 1-2-3-4 for Varying Uplink Usages. Figure 8 compares the total median whole-body dose in the building as a function of the UL usage, ranging from $0 \mathrm{~s}$ to $3600 \mathrm{~s}(1 \mathrm{~h})$ for all scenarios. It shows that, for no UL usage ( $0 \mathrm{~s}$ UL usage), scenario 4 has the highest exposure (due to the high-EIRP base stations), followed by scenario 1 (WiFi $54 \mathrm{Mbps}$ ), scenario 2 (WiFi $18 \mathrm{Mbps}$ ), and scenario 3 (UMTS). For the optimized WiFi deployments, the UL dose dominates the total dose for UL usages from less than $0.5 \mathrm{~s}$ already. For the traditional deployment for WiFi $18 \mathrm{Mbps}$, this occurs for UL usages from about $1 \mathrm{~s}$ and for the traditional deployment for WiFi 54 Mbps from about $10 \mathrm{~s}$ of UL usage. 


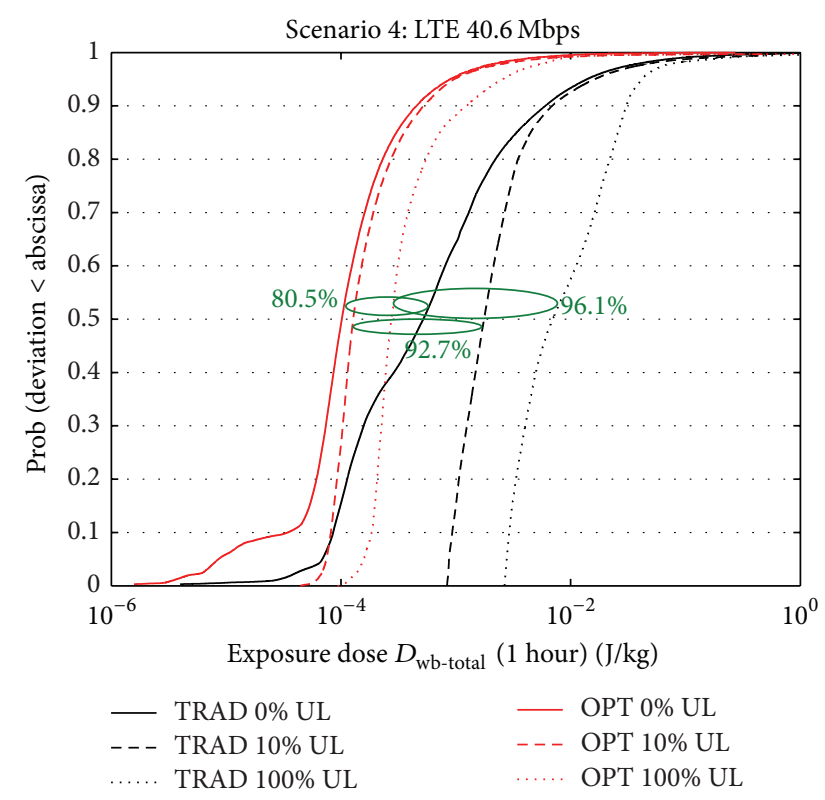

Figure 7: CDF of total dose (uplink + downlink) within one hour for scenario 4 for traditional and optimized deployment for three uplink usages $(0 \%, 10 \%$, and $100 \%$ of the time).

Eventually, all WiFi scenarios converge to a total dose that is dominated by the same UL dose, due to the same uplink power of $20 \mathrm{dBm}$. For the traditional LTE deployment, UL dominates the total dose from about $60 \mathrm{~s}$ (1 min) of UL usage. For the optimized deployment, UL becomes dominant for usages around $1000 \mathrm{~s}$ (see logscale in Figure 8). Compared to $\mathrm{WiFi}$, the total doses for LTE for high UL usages increase at a lower rate, thanks to the lower UL powers. This also counts for UMTS (scenario 3), which has the lowest doses, thanks to the lower throughput requirements and the power control mechanism.

All four scenarios show that significant exposure dose reductions can be achieved by adding more base stations with a lower transmit power. However, this comes at a higher economic cost. Future research consists of relating wholebody exposure dose due to the network to the total network installation cost [27].

\section{Conclusions and Future Work}

In this paper, total whole-body exposure doses (uplink and downlink) are jointly minimized for indoor wireless network deployments. The mathematical formulation has been given and four simulation scenarios were proposed: two $\mathrm{WiFi}$ configurations with a different throughput requirement, one UMTS voice call scenario, and one LTE high-throughput scenario. For WiFi, downlink doses are reduced by more than $95 \%$ by the optimized deployment. Due to the lack of power control, uplink usages of only a few seconds suffice to make the uplink dose higher than the downlink dose, limiting the reductions of the optimized deployment for longer uplink usages. Deployments with lower WiFi throughputs benefit less from optimizing the access point configuration. For

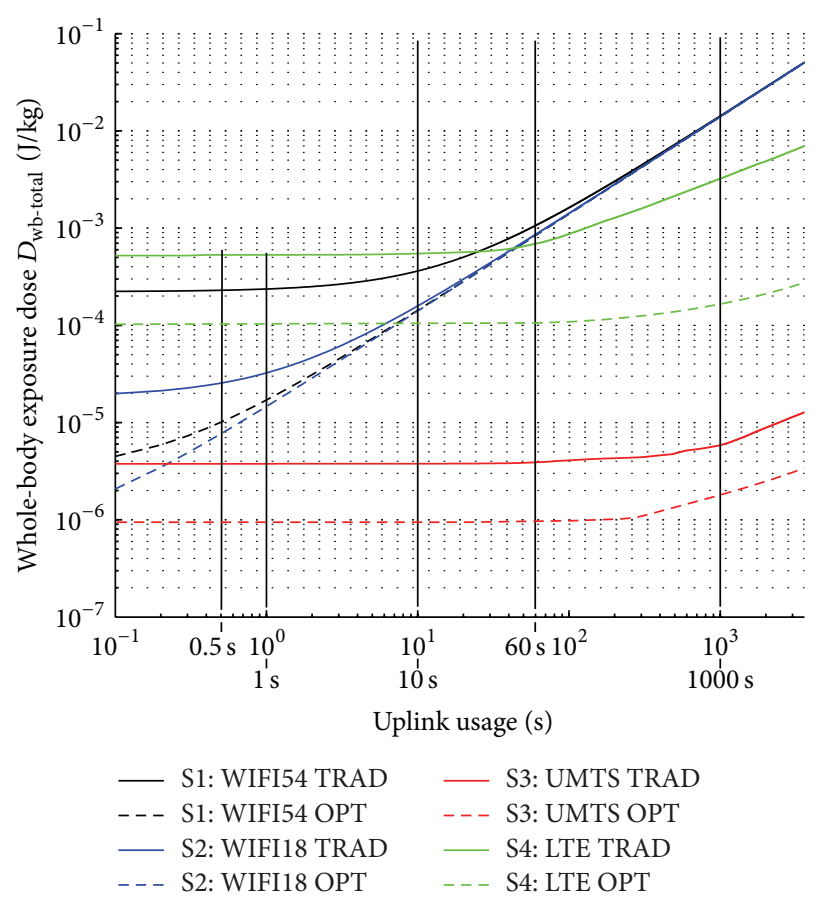

Figure 8: Whole-body total exposure dose within one hour as a function of uplink usage for the four scenarios and the traditional and optimized deployment.

UMTS, total dose reductions vary between $73 \%$ and $83 \%$, irrespective of the uplink usage, thanks to the power control mechanism. For the LTE configuration with high-power base stations, dose reductions are at least $80 \%$ and increase for higher uplink usages. For UMTS and LTE, an almost continuous uplink usage is required to induce a significant effect on the total dose, again thanks to the power control mechanism.

In future research, the influence of the uplink transmission of other users will be accounted for and localized doses will be calculated. Also, a technoeconomic analysis will be done to link the (lower) exposure to the (higher) network installation cost, and the influence of the number of users and their usage profiles on the actual duty cycle of an access point will be investigated.

\section{Conflict of Interests}

The authors declare that there is no conflict of interests regarding the publication of this paper.

\section{Acknowledgment}

This paper reports work undertaken in the context of the project LEXNET. LEXNET is a project supported by the European Commission in the 7th Framework Programme (GA n318273). For further information, please visit http://www.lexnet-project.eu. 


\section{References}

[1] IEEE Standards Association, "IEEE standard for safety levels with respect to human exposure to radio frequency electromagnetic fields, $3 \mathrm{kHz}$ to $300 \mathrm{GHz}$," IEEE Std C95.1, 1999.

[2] ICNIRP, "Guidelines for limiting exp osure to time-varying electric, magnetic, and electromagnetic fields (up to $300 \mathrm{GHz}$ )," Health Physics, vol. 74, no. 4, pp. 494-522, 1998.

[3] K. R. Foster, "Radiofrequency exposure from wireless lans utilizing Wi-Fi technology," Health Physics, vol. 92, no. 3, pp. 280-289, 2007.

[4] W. Joseph, P. Frei, M. Roösli et al., "Comparison of personal radio frequency electromagnetic field exposure in different urban areas across Europe," Environmental Research, vol. 110, no. 7, pp. 658-663, 2010.

[5] W. Joseph, P. Frei, M. Röösli et al., "Between-country comparison of whole-body SAR from personal exposure data in Urban areas," Bioelectromagnetics, vol. 33, no. 8, pp. 682-694, 2012.

[6] P. Frei, E. Mohler, A. Bürgi et al., "A prediction model for personal radio frequency electromagnetic field exposure," Science of the Total Environment, vol. 408, no. 1, pp. 102-108, 2009.

[7] A. Boursianis, P. Vanias, and T. Samaras, "Measurements for assessing the exposure from 3G femtocells," Radiation Protection Dosimetry, vol. 150, no. 2, Article ID ncr398, pp. 158-167, 2012.

[8] W. Joseph, L. Verloock, F. Goeminne, G. Vermeeren, and L. Martens, "Assessment of RF exposures from emerging wireless communication technologies in different environments," Health Physics, vol. 102, no. 2, pp. 161-172, 2012.

[9] Z. Ji, B.-H. Li, H.-X. Wang, H.-Y. Chen, and T. K. Sarkar, "Efficient ray-tracing methods for propagation prediction for indoor wireless communications," IEEE Antennas and Propagation Magazine, vol. 43, no. 2, pp. 41-49, 2001.

[10] R. P. Torres, L. Valle, M. Domingo, S. Loredo, and M. C. Diez, "CINDOOR: an engineering tool for planning and design of wireless systems in enclosed spaces," IEEE Antennas and Propagation Magazine, vol. 41, no. 4, pp. 11-22, 1999.

[11] G. Wölfle, R. Wahl, P. Wertz, P. Wildbolz, and F. Landstorfer, "Dominant path prediction model for indoor scenarios," in Proceedings of the German Microwave Conference (GeMIC '05), Ulm, Germany, April 2005.

[12] A. G. Dimitriou, S. Siachalou, A. Bletsas, and J. N. Sahalos, "An efficient propagation model for automatic planning of indoor wireless networks," in Proceedings of the 3th European Conference on Antennas and Propagation (EuCAP '10), Barcelona, Spain, April 2010.

[13] P. Sebastiao, R. Tome, F. Velez et al., "WLAN planning tool: a techno-economic perspective," in Proceedings of the COST 2100 TD (09) 935 Meeting, Vienna, Austria, September 2009.

[14] S. Phaiboon, "An empirically based path loss model for indoor wireless channels in laboratory building," in Proceedings of the IEEE Region 10 Conference on Computers, Communications, Control and Power Engineering (TENCON '02), vol. 2, pp. 1020 1023, October 2002.

[15] J. M. Keenan and A. J. Motley, "Radio coverage in buildings," British Telecom Technology Journal, vol. 8, no. 1, pp. 19-24, 1990.

[16] D. Plets, W. Joseph, K. Vanhecke, E. Tanghe, and L. Martens, "Coverage prediction and optimization algorithms for indoor environments," EURASIP Journal on Wireless Communications and Networking, vol. 2012, p. 123, 2012.

[17] D. Plets, W. Joseph, K. Vanhecke, and L. Martens, "Exposure optimization in indoor wireless networks by heuristic network planning," Progress in Electromagnetics Research, vol. 139, pp. 445-478, 2013.

[18] D. Plets, W. Joseph, S. Aerts, K. Vanhecke, and L. Martens, "Prediction and comparison of downlink electric-field and uplink localized SAR values for realistic indoor wireless network planning," Radiation Protection Dosimetry. In press.

[19] S. Aerts, D. Plets, L. Verloock, W. Joseph, and L. Martens, "Assessment and comparison of RF EMF exposure in femtocell and macrocell scenarios," Bioelectromagnetics, 2013.

[20] O. Lauer, P. Frei, M.-C. Gosselin, W. Joseph, M. Röösli, and J. Fröhlich, "Combining near- and far-field exposure for an organspecific and whole-body RF-EMF proxy for epidemiological research: a reference case," Bioelectromagnetics, vol. 34, no. 5, pp. 366-374, 2013.

[21] W. Joseph, D. Pareit, G. Vermeeren et al., "Determination of the duty cycle of WLAN for realistic radio frequency electromagnetic field exposure assessment," Progress in Biophysics and Molecular Biology, vol. 111, no. 1, pp. 30-36, 2013.

[22] ITU-R Recommendation P.1546, "Method for point-to-area predictions for terrestrial services in the frequency range $30 \mathrm{MHz}$ to $3000 \mathrm{MHz}$," 2003-2005.

[23] A. Gati, E. Conil, M.-F. Wong, and J. Wiart, "Duality between uplink local and downlink whole-body exposures in operating networks," IEEE Transactions on Electromagnetic Compatibility, vol. 52, no. 4, pp. 829-836, 2010.

[24] 3rd Generation Partnership Project (3 GPP), “Technical specification group radio access network; evolved universal terrestrial radio access (E-UTRA); physical layer procedures (release 10)," Tech. Rep. 3GPP Std. TS 36.213 V10.5.0, 2012, http://www.3gpp.org/ftp/specs/archive/36_series/36.213/36213a50.zip.

[25] P. Joshi, "Assessment of realistic output power levels for LTE devices," Tech. Rep., Lund University, Ericsson, 2012.

[26] A. Christ, W. Kainz, E. G. Hahn et al., "The Virtual Familydevelopment of surface-based anatomical models of two adults and two children for dosimetric simulations," Physics in Medicine and Biology, vol. 55, no. 2, pp. N23-N38, 2010.

[27] D. Plets, N. Machtelinckx, K. Vanhecke et al., "Calculation tool for optimal wireless design and minimal installation cost of indoor wireless lans," in Proceedings of the IEEE International Symposium on Antennas and Propagation and USNC/URSI National Radio Science Meeting, APS-1619, Memphis, Tenn, USA, 2014. 


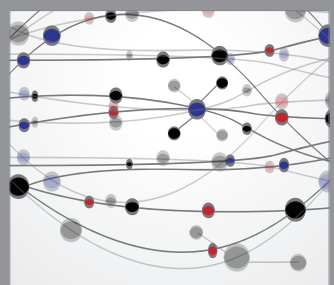

The Scientific World Journal
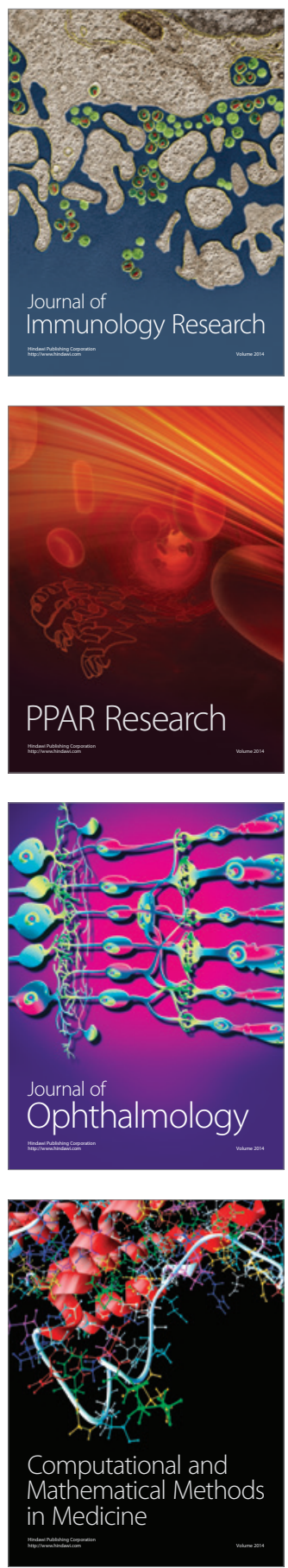

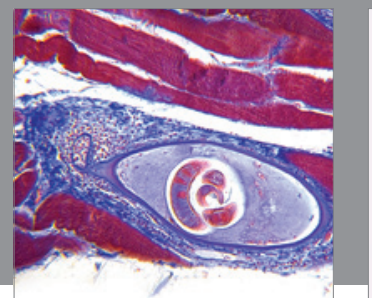

Gastroenterology

Research and Practice
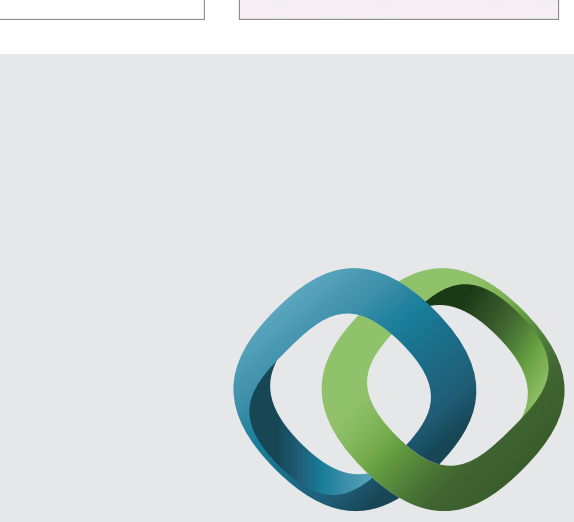

\section{Hindawi}

Submit your manuscripts at

http://www.hindawi.com
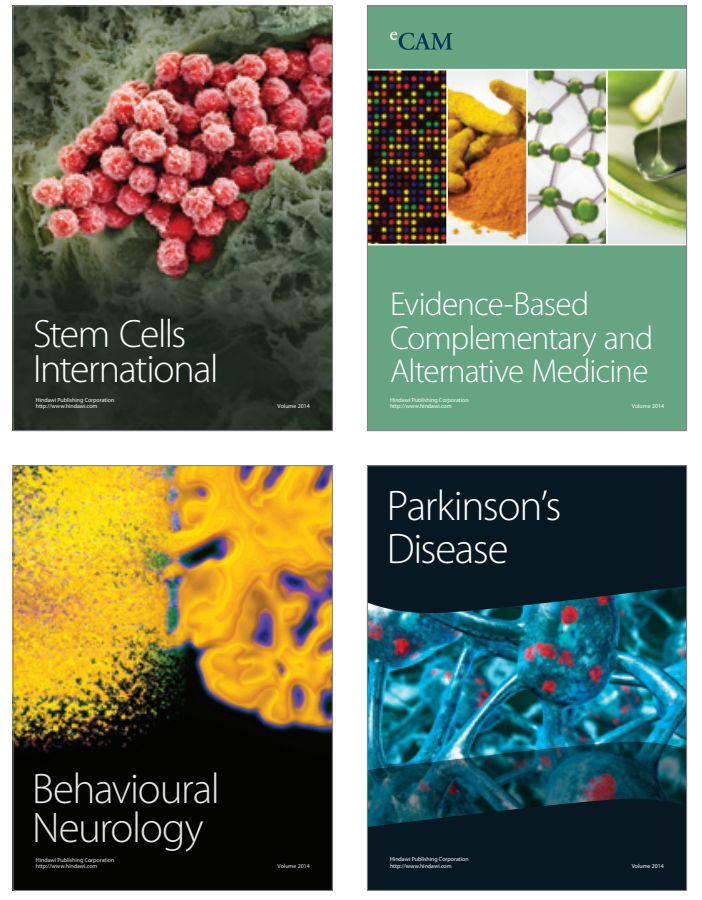
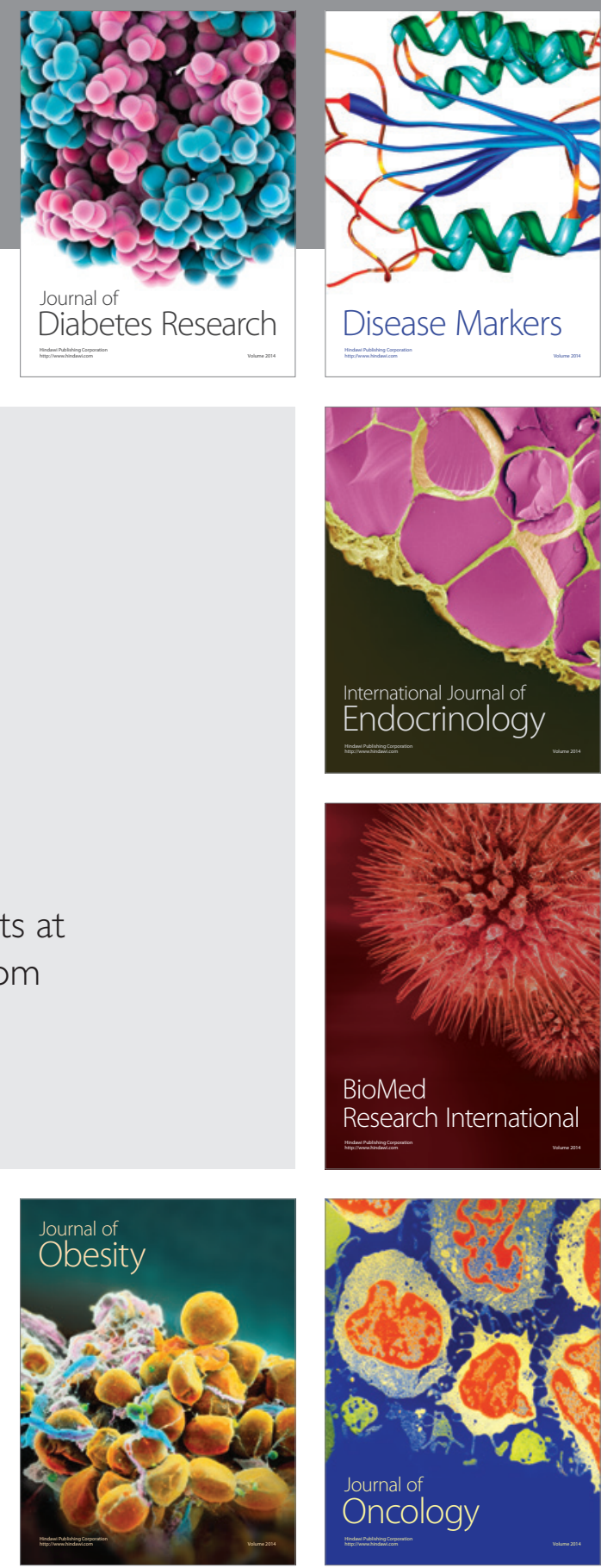

Disease Markers
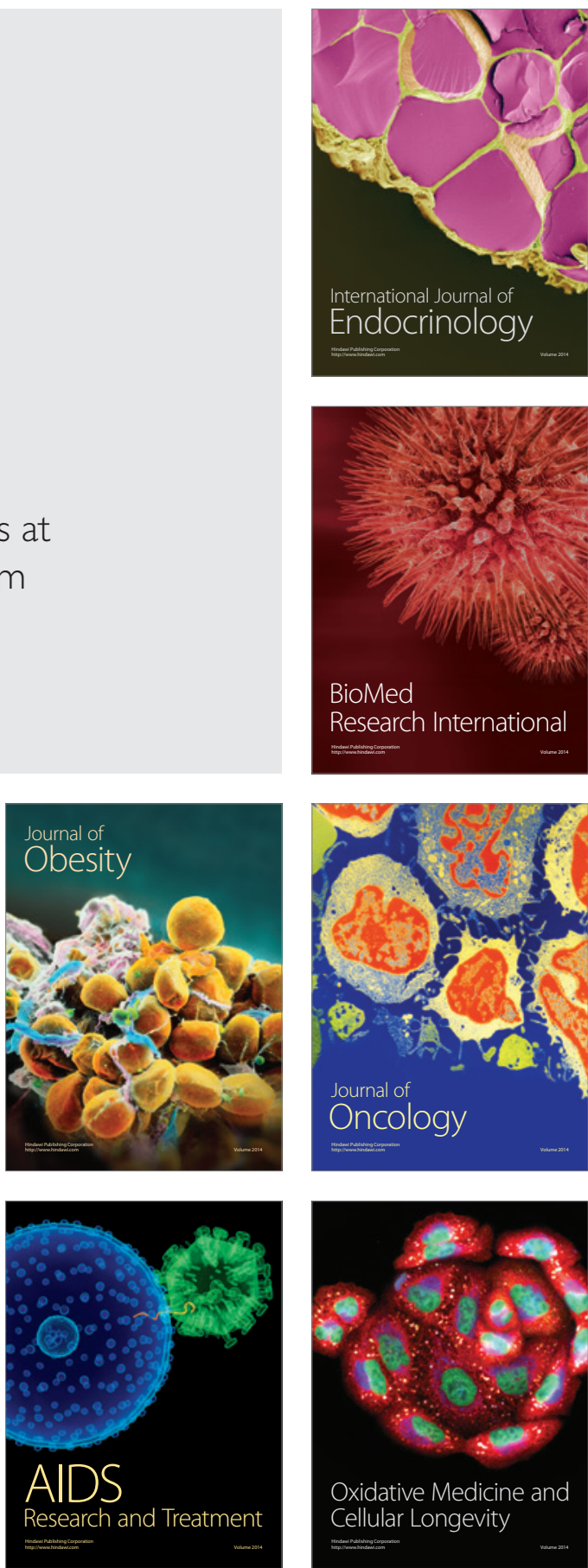